\title{
Data are in the Eye of the Beholder: Co-creating the Value of Personal Data
}

\author{
Marta Stelmaszak \\ Glenn Parry \\ London School of Economics and Political Science University of Surrey Business School \\ m.stelmaszak1@1se.ac.uk \\ g.parry@surrey.ac.uk
}

\begin{abstract}
The value of personal data has traditionally been understood in economic terms, but recent scholarship casts the value of data as multi-faceted, dynamic, emergent and co-created by stakeholders. The dynamics of the co-creation of value with personal data lacks empirical study. We conduct a case study of the development of a personalised e-book and find different perceptions of the value of personal data exist from the firm, intermediary and customer perspective: means to an end, medium of exchange and net benefit. The different data perspectives highlight ontological differences in the perception of what data are. This creates epistemological tension and different expectations of the data characteristics embedded in the process of value co-creation. The findings contribute to the growing data-in-practice literature, showing how different epistemological stances can create opposing expectations of what data should be, leading to ontological, policy and managerial tensions.
\end{abstract}

\section{Introduction}

Research into the value of data based offerings that promise improved value propositions and better utility, such as smart home solutions and internet of things, is gaining momentum [1]. The benefits of personalised solutions are contingent on generating, collecting and analysing users' personal data. While personal data data pertaining to identifiable individuals [2], [3] - are required to create personalised value offers, firms traditionally exploited data collected to further their economic gain without properly rewarding customers [4]. This resulted in an intense research focus on the economic value of personal data and redressing the imbalance of benefit distribution [3], [5].

The economic perspective may be too narrow to fully conceptualise the value of personal data. The value of data can be viewed as multi-faceted, dynamic, emergent and co-created by stakeholders [6]-[8]. Value can be perceived as a measure of 'goodness' evaluated subjectively in the eyes of external observers [9] in use [10] and in a specific context [11]. This value 'in-use' perspective suggests that value emerges during the use of a resource or a service [12], rather than 'in-exchange', typically associated with the economic view. The value-in-use perspective emphasises stakeholders' involvement in co-creating value [13], [14] and brings in their perceptions of value to the process [11]. The firm and the customer work together to co-create value [14], making value cocreation relevant in the context of personal offerings where customer-generated personal data are required for the offering to deliver its value proposition.

The question of how the perceptions of value held by stakeholders are involved in the process of cocreating the value of personal data has not been investigated in depth. The dynamics of value creation with personal data is examined here in an empirical case study of a personalised e-book, part of a 2-year publicly funded research project in the United Kingdom. Using interviews and project documentation different perceptions of the value of personal data are identified from the three main stakeholders: the firm, the intermediary, and the customer. The firm perceives the value of data primarily as means to an end rooted in the economic perception of value. The intermediary sees the value of data as a medium of exchange underpinned by the value-in-exchange perspective. Customers assess the net benefit of value, based on value-in-use. Apart from resulting in epistemological tensions, such differences bear on the ontology of data objects: stakeholders have varying and sometimes conflicting expectations of what characteristics personal data should have. This furthers the discussion on data-in-practice [6] by showing empirically that the value of data cannot, in practice, be defined from a single perspective. Policy needs to account for varying and sometimes divergent needs of stakeholders in the process of value co-creation. Finally, firms should be aware that different perspectives of value will call for different characteristics of data objects that underpin their offerings and design such offerings accordingly.

The article is structured as follows. First, we present the current literature on the value of personal data, co-creating value in use and co-creating the value of data to build towards our research question. Next, we describe research design and present the findings. We then discuss the findings and indicate implications for theory, policy and management. 


\section{Background literature}

\subsection{The value of personal data}

Personal data [2], [3] attracted renewed interest as social computing and the internet of things resulted in growth of personal data generated and collected. As research into the value of personal data developed, simultaneously concerns around privacy, consent and awareness of individuals also grew [15]. Regardless of the perspective taken, most of literature considers the value of personal data in economic terms. Personal data are invariably cast as a commodity [16], asset [5], [15], [17], good [3], property or resource [4], [18], 'new oil' [4], [15], [17] or currency [18], [19]. Firms' and individuals' interests were presented as conflicting, "a war is under way over data, but it's not entirely clear how much the resource is actually worth" [4, p. 17].

Research seeks to understand how firms capture the value of personal data by monetising them, often by exploiting data to learn about customers [15]. Studies assess data's economic value to firms by, for example, observing company stock value or revenues, tracking the prices of personal data on data marketplaces, and measuring the impact of data breaches [15].

Research adopting the individual perspective investigates how customers perceive the monetary value of their own personal data [17]. The common assumption is that customers need education as they tend to undervalue personal data [18]: "individuals do not seem to be fully aware of the monetary value of their personal data and tend to underestimate their economic power within the data-driven economy and to passively succumb to the propertization of their digital identity" [5, p. 289]. Thus, there is a plethora of research attempting to calculate the economic value of personal data for individuals [3], [5], [17].

Current literature on the value of personal data is limited by two underlying assumptions. First, a focus on economic value at the expense of other perspectives. Second, data are presented as a static resource, allowing their value to be fixed and defined. Moving beyond these two assumptions can lead to a more comprehensive understanding of the value of personal data.

\subsection{Co-creating value in use}

Value has been studied from a range of disciplines and perspectives [10]. When taken as 'goodness', value is subjectively evaluated from the perspective of an external observer [9], a phenomenological perspective
[10] where value is emergent and co-constituted by the entity and individuals within a specific context [11], [20]. Phenomenological value is thus multifaceted and difficult to define from a single perspective [11], as individuals or stakeholders interact and different ideas of value surface. The value of personal data is not embedded in data itself, it is not fixed or defined, but rather emerges from a range of perspectives, contexts, experiences and relationships brought by individuals to their interactions with data objects.

In the phenomenological view, value emerges from the interaction between the object and the observer, suggesting that "the value in objects or offerings is attributed only when individuals are able to realise their projects in their daily practices" [10, p. 211]. Named value-in-use, focus is placed on value that emerges during the use of an offering [12], deemphasising value realised in exchanges. Value-in-use involves all stakeholders in processes of co-creation: "value will have to be jointly created by both the firm and the consumer" [13], requiring understanding of interactions between customers and companies [13], acknowledging active participation as "consumers and firms can be viewed as partners in producing value during consumption" [14, p. 222].

In the context of personal data, consumers create the data using resources that may be owned by different stakeholders (phones, apps, sensors, databases etc.). Firms that derive value from personal data are not necessarily part of the consumer co-creation practice relationship. They may be secondary co-creators of meta-data generated from data on the co-creation practices of others. What emerges is a complex picture of ecosystems of value co-creation where traditional distinctions between the firm as producer and customer as consumer are blurred, and where value-in-use means the value of use of personal data for all stakeholders involved, not only the customer. The value of data is in constant flux, dependent on the use data is put to and by whom, rather than some objective measurement.

\subsection{Co-creating the value of data}

Information systems (IS) literature is moving away from conceptualising data as factual representations of the world, natural and objective in character, towards data as emergent phenomena that require unpacking [6], [21], [22]. Data are always incomplete, partial, contingent and created through "practices of conceptualization, recording and use" [6]. Jones calls for a more detailed study of how data come to be and how they are used, framing this as 'data in practice', as opposed to 'data in principle' as static entities [6]. Parmiggiani and Grisot argue that data are not finished products, but instead " a central and evolving concern 
for different stakeholders" [8, p. 2], pointing towards the role of multiple actors who co-create value. In light of limited research, the authors emphasise the need to study the dynamics of how data attain value through co-creation in settings that involve numerous stakeholders [8].

A number of studies investigate the processes of co-creating the value of data and the stakeholders involved [23], [24]. Value is a dynamic, fluid property co-created by aligning various stakeholders and their interests [7]. The value of data is shaped over time through its construction and generation, contingent upon balancing different stakeholders' concerns. In their study of data infrastructures, that is "the institutional, physical, and digital means for storing, sharing and consuming data across networked technologies" [25, p. 32] where data objects are an essential resource [26], Parmiggiani and Grisot find data generate multiple types of value for stakeholders [8]. A similar conceptualisation of the value of data is put forward by Vassilakopoulou et al. [27]. In their view, data become valuable when they are used as resources by different stakeholders, and the use value of data can be traced by identifying their role in knowledge work and task outputs [27].

IS literature highlights the need to investigate the impact of different perceptions of value on data objects generated by offerings. Current research conceptualises the value of data in different ways underpinned by varying epistemological stances, which results in often conflicting findings. To broaden understanding of the value of data, we integrate three streams of literature discussed to study how the perceptions of value held by stakeholders are involved in the process of co-creating the value of personal data.

\section{Research design}

We conducted a case study of a project to develop a personalised e-book that enhances children's reading. The UK based project - Dynamic, Real time, Ondemand Personalisation for Scaling (DROPS) received funding from EPSRC from September 2018 to August 2020. The intended output of the project is an e-book that employs personalisation technologies, collecting personal data, analysing them and providing real-time analytics that generate value for stakeholders. The project has multiple stakeholders, including publishers, small and medium enterprises, designers of personalised digital products, teachers, parents and children. To ensure that the technology is a source of value for all, the project focussed on a multistakeholder approach to value co-creation. Thus, the project and its architecture align with the research question of this paper.
The authors have been involved for the duration of the project as researchers, and participated in various design/development project meetings, and team discussions. Good, working relationships developed with all 23 stakeholders, who provided rich project documentation. The participatory nature of the authors' involvement ensured a depth of understanding of the process and product, and access to data for research purposes. One of the challenges encountered was the ongoing nature of the project during research, and the novel technology employed, contributing to the limitations of the study discussed later. Ethical approval from University of Exeter was obtained prior to project start and all participants signed relevant consent forms.

A single, representative case study [28] is a suitable source of data to support inquiry, as it allows us to uncover and analyse the various perceptions of value with respect to data held by stakeholders involved. The case study methodology is particularly effective in answering 'how' and 'why' questions and allows us to understand the phenomenon of interest where there are no clear boundaries and where context is of importance [28], [29].

Interviews were used to gather data on different value perceptions held by interviewees [29], alongside analysis of project documents. All 23 stakeholders were asked to participate and 13 agreed, with semistructured interviews lasting 25-55 minutes undertaken between January and March 2020, and subsequently transcribed. Interviewees fall into three groups: firm representatives of organisations and companies with interest in selling the final output of the project or its derivatives to potential customers; intermediary representatives of organisations responsible for developing the product and maintaining the data infrastructure; and customer - representatives of potential future customers interested in using the output product.

We collected 35 project documents, including project presentations, prototypes, photos of prototypes and diagrams drawn during meetings, meeting agendas and minutes, technical specifications of the prototype and other supplementary files. Access to all relevant documents was available as participants of the project. The summary of data collected is presented in Table 1.

\begin{tabular}{|l|l|l|}
\hline \multicolumn{3}{|l|}{ Table 1. Sources and quantities of data } \\
\hline Interviews & $\begin{array}{l}\text { Period of } \\
\text { collection: }\end{array}$ & $\begin{array}{l}\text { January to March } \\
2020\end{array}$ \\
\hline Group & $\begin{array}{l}\text { Number of } \\
\text { interviews }\end{array}$ & $\begin{array}{l}\text { Total length in } \\
\text { minutes }\end{array}$ \\
\hline Firm & 2 & 80 \\
\hline Intermediary & 6 & 244 \\
\hline Customer & 5 & 218 \\
\hline
\end{tabular}




\begin{tabular}{|l|l|l|}
\hline $\begin{array}{l}\text { Documentary } \\
\text { evidence }\end{array}$ & $\begin{array}{l}\text { Period of } \\
\text { collection: }\end{array}$ & March 2020 \\
\hline Type & $\begin{array}{l}\text { Number of } \\
\text { documents }\end{array}$ & $\begin{array}{l}\text { Total length in } \\
\text { pages }\end{array}$ \\
\hline $\begin{array}{l}\text { Meeting agenda } \\
\text { and minutes }\end{array}$ & 5 & 10 \\
\hline Presentation & 5 & 56 \\
\hline Photo & 7 & 7 \\
\hline $\begin{array}{l}\text { Project } \\
\text { documentation }\end{array}$ & 18 & 92 \\
\hline
\end{tabular}

A case study database was created in NVivo and used to conduct thematic coding [29]. We proceeded in a bottom-up manner, starting by identifying low-level codes, such as "building new products" or "improving products". Analysis focused on the practice perspective, identifying the existing and intended practices that interviewees discussed regarding the use of personal data. Emerging themes pertained to the kind of value that these practices related to, e.g. "value in use" or "educational value". Next, emerging themes were cross checked with groups of interviewees to link specific themes to particular stakeholders. Findings are presented in the next section.

\section{Findings}

\subsection{DROPS project}

The aim of the DROPS (Dynamic, Real time, Ondemand Personalisation for Scaling) project is to create decentralised models of personalisation that give benefits to organisations and individuals in a way that is privacy preserving of individuals personal data. The project acknowledges that personal data lack suitable privacy and governance structures, especially when used in product personalisation. The HAT infrastructure enables personal data to remain in the ownership of customers, permitting third party apps to undertake analysis of that data and report only metadata. E-books make use of personal data accounts (HAT Microservers provided by intermediary DataSwift.io) of each user to store data generated by users while using meta-data to personalise e-books and subsequently improve the reading experience. Storing personal data in a HAT Microserver gives full control over them to the customer, including allowing or revoking access and deleting the data. Firms can request access to these data for the purposes of personalisation, and if allowed by the user, can draw data from HAT Microservers. Findings point towards a more general complication of role division with respect to personal data. The dyad of the producer and consumer is complicated: the firm, a producer in the traditional sense, may produce the physical component of the product - the e-book - but not the data that are then used to deliver the full, personalised product. The consumer, the e-book reader, becomes a producer of data needed for the personalised product. Equally, the producer becomes the consumer of personal data produced by e-book readers. In this sense, personalised e-books are true products of value co-creation: both the firm and the customer are required to participate in the production of the personalised e-book.

The creation of the e-book and project assumed cocreation with future users and a multi-stakeholder orientation from conception "to ensure that the aspired values of e-books (for learning and privacy) are aligned with the economic models used to monetise this technology and the technical platform that delivers it". This statement acknowledges both the need to involve multiple stakeholders and that stakeholders have different perceptions of the value of data, identified pre-emptively as educational value, data privacy value and economic value.

The firms, children's book and e-book publishers (Publisher) and educational game developers (Developer), are stakeholders who will use the product under development once it is launched. The intermediaries are the HAT technology provider firms (Intermediary). The customer includes parents who would be using the e-books with their children (Parent), as well as researchers who may work on ebook data through HAT (Researcher). These stakeholder groups expressed different perspectives on the value of personal data as part of the DROPS project. Below, we present how these perceptions of value were borne out in the practices that the stakeholders discuss when engaging with this data.

\subsection{Value from the firm's perspective}

Two firms in particular were involved closely with the project: Publisher, who specialises in developing children's e-books and is keen to make use of personalisation in their products; and Developer who already uses some basic levels of personalisation in their educational games, but wished to develop this functionality further. Both firms were involved from the project beginning and remained active throughout, participating in meetings, workshops and contributing views and opinions. They perceive themselves as active participants in the co-creation process: "it's a perfect timing that we can be part of that project in the way [that] we are sharing our ideas, functionalities with what we have, can be easily useful and we can learn something new from [...] experts, that is our goal, and together we can build a new product that will help kids more" (Publisher). 
From the firm's perspective, personal data obtained through the e-book designed are valuable in two main ways. First, they are an essential component of the actual personalised product. Second, they are valuable in aggregate for running the business. In both cases personal data only gain value when they are put to use in practices, they are not inherently valuable, as described by our interviewees: "it's not really so much the data, I think, as more using it to really effectively change the experience on the fly" (Developer).

In the first case - data as an inherent component of the product - it is important for the firm to have access to personal data and be able to use them directly in the e-book. The product needs to be designed to yield the necessary data to allow for personalisation: statistics such as "most downloaded book, read only 30\%, read over $80 \%$, just downloaded, deleted on the bookshelf" were examples of data that was already available. Publisher wanted more granular data, for example identification of difficult words that children spend more time looking at in order to share this information with parents and teachers: "we can satisfy [parents'] needs in order to get them more information, personalise the information, what the kid is doing by clicking, simple clicking on, reading or answering or working on the application" (Publisher). To ensure such features are incorporated in the product, the firms contributed their knowledge of the market and pedagogy behind the product to suggest the types of data needed in the e-book. At the same time, firms were conscious that the amount of data and the intrusiveness of its collection has to be balanced with customers' needs: they quoted this as one of the reasons why they attended further workshops with the parents. This suggests that their approach was moderated by taking into account the value of data as perceived by customers. Data are valuable to the firm if they reflect the goals of the product and are traceable to specific customers who receive a personalised experience. The dominant epistemological assumption is that granular, user-level, real time reading data improve the value proposition for the customer.

In the second case, data are valuable for business development. Aggregate data help product development in response to customer preferences identified in the data: "we are doing reporting about what are the books they are downloading, what are the top downloaded books, what are the subjects and based on those downloads, we are also preparing new titles for new releases" (Publisher). Aggregate data are used to test the effectiveness of new products: "we did a small experiment [...], the book itself, it's very interesting, but it's too long and there was a lot of detail. We split the books into two parts, changed the cover and the title for that specific book, removed all the let's say not read content per page and after one week, we saw that it is in the top ten downloaded books! It was very interesting" (Publisher). Firms use aggregate data to improve existing products: "there's a lot of value for us in looking at the data on a more generic level and seeing 'half of our users struggle in this area' or 'some of our users are doing this and that', so it helps us at a macro level build better games and products and helps guide what we build and do next. Basically any data you can gather of how they're using the thing you're making is valuable" (Developer). Aggregate data are also useful for marketing purposes: "we are measuring for that market, for example, what is the target age group that they are reading more, what new content we should develop or when we should publish some books because we find that different markets, the application is visited differently" (Publisher). The same aggregate personal data are used to guide firm strategy: "we actually for this year changed our strategy, our goals, not changed but added new goals and what will be the focus" (Publisher). The large quantity of data and the possibility to aggregate them brings value to the firms. The firms recognise that this level of data contributes directly to their business goals and outcomes, and thus they acknowledge data's economic value. This is exemplified by one interviewee who identified data as a source of competitive advantage: "for us as a company, we want to be unique and we want to be in each school, that is our goal". The assumption is that high level, aggregate personal data can reveal business insights and result in competitive advantage, shaping Publisher's perception of the value of data. In both cases, the firm sees value of data as means to achieve a business outcome.

\subsection{Value from the intermediary's perspective}

The intermediary are the stakeholders who provide underpinning technology for the e-book. Referred to as Intermediary-HAT, the entities represented include the HAT platform provider (https://dataswift.io/), IP holder and governance manager, not-for-profit HAT Community Foundation (https://hatcommunity.org) and HATLAB, research, education, innovation and policy coordinator for HAT (https://hat-lab.org). The organisations are aligned in their goals and approaches to personal data and complement each other in supporting the HAT ecosystem. Representatives of Intermediary-HAT are stakeholders in the project, participating in meetings, workshops, and developing of the e-book. Their focus as intermediary is to popularise HAT Microserver personal data accounts that "mitigate the risks of managing and accessing personal data, and a richer class of information can be 
shared by users themselves". Intermediary-HAT is a key stakeholder in the product under development. Its role as an intermediary is recognised by interviewees who recognise the need for personal data among the firms and the need to protect customer data rights. "some way of reconciling those two divergent paths" (Intermediary-HAT 2).

For Intermediary-HAT, personal data are inherently valuable as a medium of exchange, hence the stated goals and mission to popularise personal data accounts in the form of HAT Microservers. Personal data are seen by Intermediary-HAT as valuable as they are something in possession of the customer that can be exchanged for something else of value provided by the firm. Intermediary-HAT firms facilitate this exchange, with its role as to "increasingly enable them [consumers] to be much more in control of this value exchange, whether it's for money, whether it's because they want to do it for social good or whether there's a less tangible benefit such as free research, free information, free service" (Intermediary-HAT 2). The intermediary sees personal data as valuable because they can be exchanged. This exchange is dependent on the capacity of data to flow: "I think a lot of people still think of data as a commodity and I think [...] it's not a commodity, it's a real time flowing beating thing [...] and so we are actually designing and creating context here to ensure that the data that flows through it can be used and valuable and of worth" (IntermediaryHAT 6). The key features of data perceived by Intermediary-HAT include their mobility and portability, dynamic character and the capacity to move between stakeholders and databases.

A number of Intermediary-HAT interviewees emphasise the idea that personal data should not be seen as a static resource, but rather as a medium of exchange: "I think it is a bit of a misnomer to be thinking of data as if it is a resource, however, data I think is much better considered to be a currency. We use data to acquire things that we value more greatly in the same way that we use money to acquire things that we value more greatly" (Intermediary-HAT 3). In the words of another interviewee, "I always use as an analogy when we talk about value, to be very careful to think about the value of data as the value of currency. When I say the value of data, I mean analogous to the value of a currency, what is the value of $\$ 10$ ? (...) Then you think about the value of your data, I think of it very much in this way so when people say, 'So how do you actually know what the value is?', I say 'I don't look at the value of data, I look at what it's being used for and then you derive from that, what is the outcome of what it's used for and where it sits in the worth of that outcome' (...) When you say 'what is the value of data?', you are saying what is the value of a store or value and a medium of exchange because data is a medium of exchange, it does not have a store or value" (Intermediary-HAT 6). In order to gain value as a medium of exchange, data need to attract several attributes shared by all media of exchange. Namely, they have to be trusted and reliable, stored in a secure way, and seen as valuable to both parties of the exchange: "what data really is, is a store value and it's then up to the individuals or the persons who then see the data and what value they can derive from it. So at HAT, our core is designed to give you a place to store that value" (Intermediary-HAT 5). Intermediary-HAT engages in trust-building initiatives with the firms and customers, implements clear data governance policies and emphasises the issues of informed consent and full control over data. At the same time, Intermediary-HAT invests in educating customers regarding the value of their personal data. This suggests that the intermediary is aware that the perceptions of value of data held by both sides of the exchange can impact the value of data as medium of exchange.

The intermediary perspective, perhaps a product of the mediating role between two sides of the exchange, rests on the assumptions that personal data are seen as a valuable medium of exchange by both parties and that they can be exchanged for other things of value. While firms seem to be aware of the capacity of data to generate value, customers are less clear in their understanding of the role of data as a medium of exchange and thus their potential value.

\subsection{Value from the customer's perspective}

Several groups are interested in the e-book, including schools, teachers, and even governments. For the purposes of this study, within the scope of the DROPS project only two groups are considered. First are parents who buy and make decisions about the use of the product, and who generate personal data about themselves and their children when reading e-books. Parents were involved on three different occasions in the evolution of the product: they were interviewed regarding the shared reading practices with their children and subsequently they participated in two prototyping workshops. Their views and opinions informed the design of the product and the underlying data. Second, since DROPS is a publicly funded research project and HAT aims to make data available for research purposes, we identify researchers who belong to Intermediary-Team as another group of customers for personal data. Researchers were developing analytics functions, assessing the business and legal viability of the product, contributing to project meetings and product development. 
Parents interviewed expressed mixed views regarding the usefulness of the e-book data: "As a parent, [...], maybe recommending books like if he enjoyed reading a book, what might my son, my children enjoy reading next, I would appreciate that but in terms of how much time we spent on something as a parent, it wouldn't really be of interest" (Parent 1). The general view expressed emphasised that the parent knows most about the child's reading practices and any associated issues from observation and additional data would be of limited use, contrary to what Publisher stipulated. It was felt that time spent with children was already scarce, and the parent would rather spend more time engaging in the activity of reading than analysing reading data: "it depends on the level of how much it interferes with that actual activity. If it's just an e-book and tracks things like not recording our conversation, not interfering with what we are doing, I think that's fine, I think for me, it becomes a problem when it tries to interfere and it also tries to change something that might be an activity that's going well otherwise" (Parent 1). Personal data about reading practices could be seen to undermine the value of time spent together during an activity, which is at odds with the sentimental value attached to shared reading practices. Parents preferred personal data collection to be as inconspicuous and limited in its scope as possible and be strictly consent-based and opt-in. This view seems to indicate that customers enjoy the functionality that data may bring, but they approach it using a calculus, weighing the benefits and costs to identify net benefit.

The granular, individual-level data were also seen as a source of issues: "it can become a bit competitive, at least it's the type of environment where my children are it's educating these parents, middle class parents and it's always this danger you start comparing. [...] Thinking more about this, the problem is not the children, I think if the children have access to what other children are reading, not so much how much but what they're reading is a good thing but I think if parents start getting involved, it could become a bit of a competition or you feel bad because you might not be reading as much as other parents, and I would be careful with that." (Parent 2). In general, the less granular the data, the more the parents were open towards collecting them when using the e-book.

However, Parent 1 was particularly interested in the potential of aggregate data to formulate recommendations: "as a parent I would love to know what some of the parents who tend to have similar tastes, what are the books they thought were suitable for their kids or whether they enjoyed it, so I would also be very happy to receive recommendations from other parents or see how they rated books" (Parent 1). The interviewee expressed several times the benefit of having useful recommendations drawn from the aggregate data and their value for educational purposes. Parents were open to share personal data with other users as long as they were anonymous. From this perspective, data need to be both portable and relational to other datasets. Aggregate personal data can reveal useful trends concerning groups of similar backgrounds and ages and contribute to the educational development and growth, thus emphasising the value of data in their use as long as this value is aligned with intended uses.

Researchers that we interviewed focussed on the value that personal data brought them for research and career purposes. They acknowledged that access to personal data at scale offered possibilities to conduct interesting and sought-after research: "researchers have to do [a lot] to collate the data but if we can get the publisher to work with us and share that data with us, then we could get a larger amount of data and based on that, to do further analysis" (Researcher 1) and "if we don't have data, we can't do empirical analysis, which is the point" (Researcher 1). The HAT technology was seen as a way to provide ethical access to data needed for research. Researchers suggested that research underpinned by personal data may be better received by journal editors, furthering their career progression. Accessible, ethically sound personal data were identified as the key value features for this group of customers. This view was based on the assumption that large amounts of quantitative data can yield research insights, giving a net benefit.

From the findings, the representatives of the firm, the intermediary and the customer exhibit different perceptions of value of personal data. These perceptions come to bear upon the co-creation of value: the process invites and encourages the stakeholders to express their ideas of what makes ebook personal data valuable through meetings, workshops and prototyping. This is evident from meeting minutes when the customer input is taken into account in the design process and discussed together with the firm: "Researcher 1: [we are] working on ebook space from a child and parent perspective; we start with design exploration. Prototyping with our two collaborators [Publisher and Developer]". Differing perceptions of value held by the project groups are accounted for in the co-creation process.

\section{Discussion}

The findings presented above show the perceptions of value held by three main stakeholder groups within a personal data related project and how these perceptions become embedded in the value co-creation 
process. Notably, the firm sees data as a means to an end: if use of data with the right attributes leads to desirable business outcomes, then data are valuable. For the intermediary, data are valuable as a medium of exchange, so they generate value when they can function in this capacity and facilitate the exchange. The customer perspective focuses on the net benefit that arises from data: the value of data results from a favourable comparison of the expected benefit with the associated costs. While all rooted in the value-in-use perspective, these perceptions have different epistemological underpinnings and lead to tensions around data. We also show how these perceptions of value become involved in the development of the final product and influence the characteristics of data.

While current literature focuses on the economic value of data [3], [5], [17], personal data are seen as valuable in different ways. Firms focus primarily on economic value and through this lens data become valuable when they can address business objectives. This can be understood as the realisation of the firm's ultimate goal: achieving its goals and putting in practice its business strategy. Since firms are primarily motivated by achieving profit, their predominant focus on the economic value of data is well placed and justified. However, for the intermediary personal data are valuable because they can function as a medium of exchange. This is aligned with the nature of the intermediary's role: they exist as facilitators between the firm and the customer and they can only generate and capture value if they support some kind of an exchange between the two parties. The customer emphasises the value of personal data that is a result of the net benefit. Again, this is a common mechanism in assessing value by individuals: in all kinds of exchanges, customers will focus on their own net benefit calculus as it is most directly linked to the value they receive. With three stakeholders and a single case study, we show that there are at least three different perceptions of value that come together in the co-creation of a single offering.

These perceptions of value, while not always in direct conflict, can lead to tensions between stakeholders. The value embedded in the final offering results from negotiations and trade-offs between stakeholders to ensure that the outcome satisfies various needs. The value of personal data is not static, defined or fixed, but is a product of changing needs and contexts that put the value of personal data in flux, and makes product development a challenge. Returning to the analogy used by one of our interviewees, trying to calculate the value of personal data is much like asking how much $\$ 10$ is worth, which is near impossible to answer without taking the wider context into account and accepting the fact that worth fluctuates over time.

Our research shows that in the context of personalised offerings the distinction between the producer and the consumer is blurred. The firm, as the producer, supplies parts of the product necessary for the realisation of value, but the customer as consumer of the product in equal parts co-creates the value of the offering. Without personal data generated by the customer, the product would not be complete, or even functional. Without data, the product is just a standard e-book. Literature acknowledges that both the producer and the consumer have to be involved in the cocreation of value to maximise the benefits [13], [14].

In data based offerings involving personalisation, the value of such offerings can only ever be realised in their use: personal data generated through using the product are necessary to personalise it and thus generate the stipulated value. The increasingly complex ecosystems of value emerging around personal data requires the creation of policies, governance and trust around how data can be used. Equally, it is the intermediary who becomes charged with facilitating and managing the negotiation of value from different perspectives between various stakeholders involved in the value co-creation processes. An intermediary who sees the value of personal data from the medium of exchange perspective contributes to the creation of value by providing a fair and neutral mechanism for data to be continuously exchanged.

Our investigation of the data value co-creation process shows that different stakeholders bring in different perspectives of value of personal data, confirming and extending the propositions put forward in existing literature [6]-[8]. These differences bear not only upon the expectations of value, but also influence the shape of data. From the economic perspective of the firm, data become valuable through their granularity and traceability to individual customers on one hand, and quantity and aggregate character on the other. As a medium of exchange, data need to be mobile, portable, of high quality and characterised by trustworthiness. To realise their net benefit value, data need to be at aggregate level, relational, ethical and obtained with appropriate consent. There is a clear link between the less concrete perceptions of value and the tangible characteristics of data. The process of cocreating the value of data will influence not only how data are used, but also how data are [6]. The plurality of the desired characteristics of data brings with it added complexity, and while not mutually exclusive, some of these characteristics are at odds with each other. 
Our findings have several implications, notably for theory, policy and management. From the theoretical perspective, research shows that different perceptions of value of personal data, underpinned by differing epistemological stances, result in tensions and varying expectations around data that are created and used. The means to an end perspective of the firm justifies the fit between data and desired business outcomes, guided primarily by the economic perspective. This is at odds with the net benefit calculus approach of the customer where the data collection and processing costs need to be lower than the perceived benefit of data. The intermediary is dependent on data flow, and so benefits if both sides of the exchange have a coherent perception of the value of data.

We provide clarity of the epistemological value tensions to be managed. Firms identify valuable data as granular, real time, user level and in large volume. Customers expect data to be ethically collected, inconspicuous, narrow in scope, anonymized and generic. The potential tensions these differences create may be addressed by the intermediary. The co-creation process entails careful management of epistemologies via manipulation of the offering to facilitate cocreation of value that addresses both perspectives.

More research is needed to uncover the different ways in which personal data can be perceived as valuable, aside from the two other perceptions identified in this paper. Similarly, shifting the perspective on personal data from a static resource to a dynamic "currency" can, with the help of further research, yield important findings in this area. The fundamental value-in-use of personalised offerings opens a new perspective on value co-creation of databased products and calls for a further investigation of the role of the consumer as producer in the context of personalisation. The role of intermediaries and the medium of exchange perspective on personal data require further study. Further research is needed to gain a better understanding of how the various perspectives on the value of personal data influences the characteristics of data objects when they are cocreated.

Policy around personal data needs to broaden in scope beyond protecting the interests of various stakeholders, most notably the customer. Policy and governance need to focus on ensuring that the varying and sometimes divergent needs of stakeholders that converge in data objects are met. This requires putting in place policies, rules and processes that ensure equal participation and involvement of stakeholders in the co-creation of personalised offerings. In this sense, policy shapes value at the macro level, and the intermediaries implement policy mechanisms in practice at the meso level.
Finally, our findings indicate that managers need to ensure that personalised offerings align the value propositions of all stakeholder value perceptions. Different perceptions of value call for data with different characteristics. Appreciating the dissonance in the perceptions of value followed by a careful alignment of value proposition with value perceptions may lead to better offerings and consequently competitive advantage.

\section{Conclusions}

In this paper, we show how the perceptions of value of personal data held by stakeholders become involved in the process of value co-creation in the context of a data based personalised offering. The perceptions of value of personal data as a means to an end held by the firm, medium of exchange for the intermediary, and net benefit for the customer create tensions in the cocreation process and result in sometimes divergent expectations of the characteristics of data. We contribute to existing literature by empirically showing that data are not neutral, objective, referential or natural [6], but instead they are a result of a complex process of negotiation and co-creation of value understood from different epistemological perspectives. We show how differences in epistemology between stakeholders impinge on the ontology of data objects and shape their characteristics. Thus, we connect the idea of the value of data with their characteristics and show in what way these characteristics, such as granularity, portability, veracity or relationality, depend on the conceptualisations of value.

Our findings are limited by the nature of the case study we analysed and its context. As a publicly funded research project, the process of co-creation concerned a particular offering and operated without typical market constraints. The data based personalised e-book was still in development when we conducted our study. Thus, more research is needed in more common contexts to further our understanding of how the perceptions of value shape the ontology of data objects in different contexts and areas. Further studies should investigate the principles of developing data based personalised products while acknowledging the differences in value perceptions around data. Finally, more research is required around governance and policy to understand how to best develop offerings that answer the sometimes divergent needs around personal data.

\section{References}


[1] G. C. Parry, S. A. Brax, R. S. Maull, and I. C. L. Ng, "Operationalising IoT for reverse supply: the development of use-visibility measures," Supply Chain Manag., vol. 21, no. 2, pp. 228-244, 2016.

[2] N.d., "What is personal data?," Information Commissioner's Office, 2020.

[3] M. Chessa and P. Loiseau, "A cooperative game-theoretic approach to quantify the value of personal data in networks," Proc. NetEcon 2017 12th Work. Econ. Networks, Syst. Comput. - Conjunction with ACM EC 2017 18th ACM Conf. Econ. Comput., vol. 3, no. 2014, p. 2015, 2017.

[4] L. Kugler, "The war over the value of personal data," Commun. ACM, vol. 61, no. 2, pp. 17-19, 2018.

[5] G. Malgieri and B. Custers, "Pricing privacy - the right to know the value of your personal data," Comput. Law Secur. Rev., vol. 34, no. 2, pp. 289-303, 2018.

[6] M. Jones, "What we talk about when we talk about (big) data,” J. Strateg. Inf. Syst., vol. 28, no. 1, pp. 3-16, 2019.

[7] M. Barrett, E. Oborn, and W. Orlikowski, "Creating value in online communities: The sociomaterial configuring of strategy, platform, and stakeholder engagement," Inf. Syst. Res., vol. 27, no. 4, pp. 704-723, 2016.

[8] E. Parmiggiani and M. Grisot, "Data infrastructures in the public sector: A critical research agenda rooted in Scandinavian research," in 10th Scandinavian Conference on Information Systems (SCIS), Nokia, Finland, 2019, pp. 1-16.

[9] J. F. Moore, "Predators and Prey: A New Ecology of Competition," Harv. Bus. Rev., vol. 71, no. 3, pp. 75-86, 1993.

[10] I. C. L. Ng and L. A. Smith, "An integrative framework of value,” Rev. Mark. Res., vol. 9, pp. 207-243, 2012.

[11] M. A. Akaka and G. Parry, "Value-in-Context: An Exploration of the Context of Value and the Value of Context," in Handbook of Service Science, Volume II, Service Science: Research and Innovations in the Service Economy., vol. II, P. P. et al. Maglio, Ed. Springer Nature Switzerland AG, 2019, pp. 457-479.

[12] S. L. Vargo and R. F. Lusch, "Service-dominant logic: Continuing the evolution," J. Acad. Mark. Sci., vol. 36, no. 1, pp. 1-10, 2008.

[13] C. K. Prahalad and V. Ramaswamy, "Co-creation experiences: The next practice in value creation," J. Interact. Mark., vol. 18, no. 3, pp. 5-14, 2004.

[14] R. L. Priem, "A consumer perspective on value creation,” Acad. Manag. Rev., vol. 32, no. 1, pp. 219-235, 2007.

[15] M. van Lieshout, “The Value of Personal Data," IFIP
Adv. Inf. Commun. Technol., no. May 2015, pp. 3-16, 2015.

[16] P. M. Schwartz, "Property, Privacy, and Personal Data," Harv. Law Rev., vol. 117, no. 7, pp. 2056-2128, 2004.

[17] S. Spiekerman and J. Korunovska, "Towards a value theory for personal data," J. Inf. Technol., vol. 32, pp. 62-84, 2017.

[18] C. Gates and P. Matthews, "Data is the new currency," ACM Int. Conf. Proceeding Ser., pp. 105-116, 2014.

[19] O. Rana and J. Weinman, "Data as a currency and cloud-based data lockers," IEEE Cloud Comput., vol. 2, no. 2, pp. 16-20, 2015.

[20] J. D. Chandler and S. L. Vargo, "Contextualization and value-in-context: How context frames exchange," Mark. Theory, vol. 11, no. 1, pp. 35-49, 2011.

[21] L. Gitelman and V. Jackson, "Introduction," in "Raw Data" is an Oxymoron, L. Gitelman, Ed. Cambridge, MA: MIT Press, 2013, pp. 1-14.

[22] I. D. Constantiou and J. Kallinikos, "New games, new rules: Big data and the changing context of strategy," J. Inf. Technol., vol. 30, no. 1, pp. 44-57, 2015.

[23] W. A. Günther, M. H. Rezazade Mehrizi, M. Huysman, and F. Feldberg, "Debating big data: A literature review on realizing value from big data,” J. Strateg. Inf. Syst., vol. 26, pp. 191-209, 2017.

[24] R. Sharma, S. Mithas, and A. Kankanhalli, "Transforming decision-making processes: a research agenda for understanding the impact of business analytics on organisations," Eur. J. Inf. Syst., vol. 23, no. 4, pp. 433-441, 2014.

[25] R. Kitchin, The Data Revolution. Big Data, Open Data, Data Infrastructures and Their Consequences. London: SAGE Publications, 2014.

[26] C. Alaimo, J. Kallinikos, and E. Valderrama-Venegas, "After social media: the formation of the Tripadvisor platform ecosystem,” J. Inf. Technol., pp. 1-40, 2020.

[27] P. Vassilakopoulou, E. Skorve, and M. Aanestad, "Enabling openness of valuable information resources: Curbing data subtractability and exclusion," Inf. Syst. J., vol. 29, no. 4, pp. 768-786, 2019.

[28] R. Yin, Case study research. Design and Methods. London: Sage, 1994.

[29] C. Robson, Real world research: a resource for users of social research methods in applied settings. Padstow: John Wiley, 2011. 